\title{
Assessment of sewage sludge bioremediation at different hydraulic retention times using mixed fungal inoculation by liquid-state bioconversion
}

\begin{abstract}
Sustainable, environmental friendly, and safe disposal of sewage treatment plant (STP) sludge is a global expectation. Bioremediation performance was examined at different hydraulic retention times (HRT) in 3-10 days and organic loading rates (OLR) at 0.66-7.81 g chemical oxygen demand (COD) per liter per day, with mixed filamentous fungal (Aspergillus niger and Penicillium corylophilum) inoculation by liquid-state bioconversion (LSB) technique as a continuous process in large-scale bioreactor. Encouraging results were monitored in treated sludge by LSB continuous process. The highest removal of total suspended solid (TSS), turbidity, and COD were achieved at 98, 99, and 93\%, respectively, at 10 days HRT compared to control. The minimum volatile suspended solid/suspended solid implies the quality of water, which was recorded 0.59 at 10 days and 0.72 at 3 days of HRT. In treated supernatant with $88 \%$ protein removal at 10 days of HRT indicates a higher magnitude of purification of treated sludge. The specific resistance to filtration (SRF) quantifies the performance of dewaterability; it was recorded minimum $0.049 \times 1012 \mathrm{~m} \mathrm{~kg}-1$ at 10 days of HRT, which was equivalent to $97 \%$ decrease of SRF. The lower OLR and higher HRT directly influenced the bioremediation and dewaterability of STP sludge in LSB process. The obtained findings imply encouraging message in continuing treatment of STP sludge, i.e., bioremediation of wastewater for environmental friendly disposal in near future.
\end{abstract}

Keyword: Bioconversion; Dewaterability; Filamentous fungal inoculation; Hydraulic retention time; Organic loading rates; Wastewater sludge 